\title{
EDITORIAL
}

\section{The life and times of the nucleus}

\author{
Yamini Dalal $^{1}$
}

Published online: 30 November 2021

This is a U.S. government work and not under copyright protection in the U.S.; foreign copyright protection may apply 2021

Wandering the halls of the Palace of Versailles one balmy summer day during my sabbatical in Europe a few years ago, I stumbled upon a 1750 s microscope crafted by the French optician Claude Passemant, encased in a display cabinet in an antechamber of Louis XV's royal chambers. It was tempting to imagine the King peering down the barrel of the scope, wondering with curiosity what to make of the miniscule objects he may have seen magnified, for which theories of that time had nascent explanation. From the earliest days of microscopic observation of living organisms by Hooke and others in the $1660 \mathrm{~s}$, to the iconic structure of chromosomes - telomeres, arms, and centromeres reported later, the faithful inheritance of genetic information and its relationship to the strikingly distinctive structures seen under the microscope have captivated scientists.

Chromosoma was founded in 1939, establishing itself in the midst of two world wars as the place to publish seminal studies of chromosomes and the nucleus. The first articles of the journal were published in German (some were even written in German by non-German scientists) and brought to light the sub-structure of chromosomes in various organisms analyzed by zoologists and cytogeneticists. Since that time, Chromosoma has published $\sim 5300$ scholarly advances dissecting how the genome is organized and functions (or, is disorganized and does not function) across a range of species, with concurrent development of tools that did not exist before. Some of these landmark studies have been cited thousands of times, and are listed in an elegant editorial written some years ago by the former Editor in Chief Erich Nigg on the 75th year anniversary of the journal (1). These discoveries are profound; they encompass every part of the nucleus - chromosome bands, centromere structure, telomeres, the nuclear lamina, regulation of transcription and translation, splicing, repair, replication, post-translational modifications, molecular motors that control and modify

Yamini Dalal

eicchromosoma@gmail.com

1 Center for Cancer Research, NCI, NIH, Bethesda, MD, USA the genome, and cell-cycle specific chromosome dynamics in mitosis and meiosis in single and multicellular organisms. Remarkably, this comprehensive body of work originates from labs around the globe, reflecting the universal appeal of the subject. Even in the face of societal and situational differences, that primal human curiosity to understand the basis of life remains a unifier and driver of scientific endeavors.

After serving for 10 years on the board, I have the honor and privilege to succeed Dr. Simon Boulton as Editor in Chief of Chromosoma. My service follows in a long line of distinguished Editors in Chief, whose footsteps will be hard to fill! In a sign of just how diverse chromosome biology is as a field, I am the first woman, first person of color, first immigrant Indian-American Editor in Chief of this prestigious journal. My fascination for all things associated with chromosomes dates back to when I was a child, transfixed by a chapter on chromosomal abnormalities in a textbook on human medicine. I still recall being amazed by syndromes in which people had extra chromosomes, rather naively imagining it might imbue them with superpowers. This early exposure to the power of chromosomes turned into a life-long obsession to learn about their unit structure, their organization, dynamic behavior, function, and dysfunction.

My vision for the journal, building upon the legacy of the luminaries who preceded me, is very simple. We want to publish discoveries which answer outstanding questions and illuminate phenomena in nuclear biology that have remained hidden. For example, what is the role of force and mechanics in 3D dynamics of chromatin? How are chromatin states renewed across different developmental points and at breakpoints? How does 3D organization or function alter with age, disease, and across evolution? What is the role of the "dark" repetitive matter of genomes, which comprises the vast majority of DNA, in the functional and evolutionary context? How do noncoding RNAs effect epigenetic fate, or even cell lineage? How do topological knots get resolved? Why don't loops collide in real time in the nucleus? Why are some organisms more sensitive to ploidy changes than others? How does the cell communicate signals from the external world to titrate gene expression or chromatin shape 
inside the nucleus? How do mitosis and meiosis change chromosome structure and vice versa? How do translocations occur in real time? Does epigenetic fate hinge upon the local chromatin fiber context? How is transcription and translation coupled to the epigenetic state of the gene? What are the cutting-edge tools needed to probe these mysteries of the genome across scales, in living cells, in tissues, and in non-model organisms? This is by no means an exhaustive list of questions, but only a glimpse into the many avenues for exploration within the nucleus.

To this end, we will be examining these topics in special collections, headed by expert guest editors and editorial board members, in the upcoming 3 years. We invite original work that focuses on any of these, and related areas. We promise a constructive process to help get your science disseminated in publication. Where possible, we encourage transfers of positively reviewed papers of importance and interest from other journals that may be lacking in mechanism, but which possess provocative conceptual advances, and experimental rigor. We especially welcome new investigator submissions. To inspire our next generation of scientists, we will be sharing "Coffee and Conversations" with editors, to illuminate the myriad journeys that lead to exceptional science and mentorship. Finally, to recognize the efforts of undergraduate, graduate student, and postdoctoral investigators who are the future of science, we have reinstated the "Chromosoma Award." This award, selected by an editorial jury every December, will go to the best Chromosoma paper in that year which makes a breakthrough on an important question in the field.

On behalf of our newly expanded editorial board, with experts representing every facet of the nucleus, and from countries all around the world, it is with an egalitarian spirit that we welcome you to submit your exciting and rigorous discoveries to Chromosoma.

Publisher's note Springer Nature remains neutral with regard to jurisdictional claims in published maps and institutional affiliations. 